\title{
In situ measurement of leaf water use efficiency of lilac (Syringa vulgaris): comparison with crop plants
}

\author{
O. Bethenod, J. Pilarski ${ }^{\star}$ and P. Quetin
}

INRA, Station de Bioclimatologie, 78850 Thiverval-Grignon, France

\section{Introduction}

In order to understand the regulation between the net $\mathrm{CO}_{2}$ assimilation rate (A) and the transpiration rate $(E)$, leaf gas exchange was measured in the field; leaf water use efficiency (WUE) of lilac (Syringa vulgaris) was compared to those of maize (Zea mays L.) and potato (Solanum tuberosum L.).

Bierhuizen and Slatyer (1965) pointed out that, for a given water saturation deficit (vpd), WUE (A/E), at the leaf level depends upon the intercellular $\mathrm{CO}_{2}$ concentration $\left(C_{i}\right)$ and stomatal conductance $\left(g_{\mathrm{c}}\right): A=g_{\mathrm{c}}\left(C_{\mathrm{a}}-C_{\mathrm{i}}\right) ; E=1.6 g_{\mathrm{c}}$ $(v p d) ; \quad A V=\left(C_{\mathrm{a}}-C_{\mathrm{i}}\right) / 1.6 \quad v d p ; \quad$ with $C_{\mathrm{a}}=\mathrm{CO}_{2}$ concentration in air.

A direct estimate of WUE is therefore given by the slope of the relationship between $A$ and $g_{c}$. All 3 species considered here are able to maintain their xylem water potential: regardless of the value their predawn water potential reached between 0.2 and $0.6 \mathrm{MPa}$, the minimal xylem water potential did not fall below $-1.3 \mathrm{MPa}$ for potato and $-1.6 \mathrm{MPa}$ for maize and lilac at Grignon. In this case, $C_{i}$ remains constant throughout the day (Bethenod et al., 1988).

Jones (1973) proposed to represent this regulation by the curve of $A$ versus $C_{i}$ called the demand function (Farquahar and Sharkey, 1982). If $C_{a}$ is placed on the $C_{\mathrm{i}}$ axis, the leaf $\mathrm{CO}_{2}$ conductance $\left(g_{\mathrm{c}}\right)$ is the slope of the straight line joining $C_{\mathrm{a}}$ to the corresponding $C_{i}$ on the demand function: this defines the supply function. Our first aim was to study the proportionality between $A$ and $g_{c}$, in order to show how demand function and supply function adjust to each other. But beyond a limit on the demand function, $C_{i}$ increases and WUE decreases because of large $g_{\mathrm{c}}$ values; $A$ then remains at its maximal value $\left(A_{\max }\right)$. The second aim of this work was to compare the $A_{\max }$ values for the studied species.

\footnotetext{
${ }^{\star}$ Present address: Polish Academy of Sciences, Laboratory of Photosynthesis, St Jana 22, 31-018 Cracow,
} Poland. 


\section{Materials and Methods}

Lilac, potato and maize were grown in the field at Grignon, $40 \mathrm{~km}$ west of Paris.

Measurements were made with a Parkinson leaf chamber (A.D.C.). The gas circuit was modified: pressurized dry air from cylinders provided a $\mathrm{CO}_{2}$ concentration in the chamber higher than that in natural air. Two gas-flow controllers (Tylan) ensured a constant flow rate at both reference and chamber levels.

$\mathrm{CO}_{2}$ net assimilation (A) data were normalized at $338 \mu \mathrm{mol} \cdot \mathrm{mol}^{-1}$ for $C_{2}$, according to Bethenod et al. (1988) for $C_{3}$ leaves; for $C_{4}$ leaves, $A$ is approximately the same above 320 $\mu \mathrm{mol} \cdot \mathrm{mol}^{-1} \mathrm{CO}_{2}$. Fig. 1 shows 3 hypothetical adjustments between demand and supply functions. The data shown in following figures correspond to a typical day for each species. Each symbol represents a leaf on different plants in the field for maize and potato, and of 2 trees in a hedge for lilac.

\section{Results}

Normalized net assimilation $\left(A_{c}\right)$ is plotted versus photosynthetic photon flux density $(P P F D)$ in Fig. 2. Note that the lilac data show a low scatter. For potato, the high scatter could indicate water stress; but this is not apparent from leaf water potential data (Bethenod et al., 1988). This scatter can be induced by: 1) individual variability and 2) changes in $A$ between morning and evening at the same level of incident $P P F D$. The maximum values for potato are about the same as those for lilac.

$C_{\mathrm{i}}$ increases slightly when PPFD decreases below $500 \mu \mathrm{mol} \cdot \mathrm{m}^{-2} \cdot \mathrm{s}^{-1}$ (Fig. 3).

Fig. 4 displays $A_{c}$ versus $g_{c}$. Up to $g_{\mathrm{c}}$ values between 0.20 and 0.23

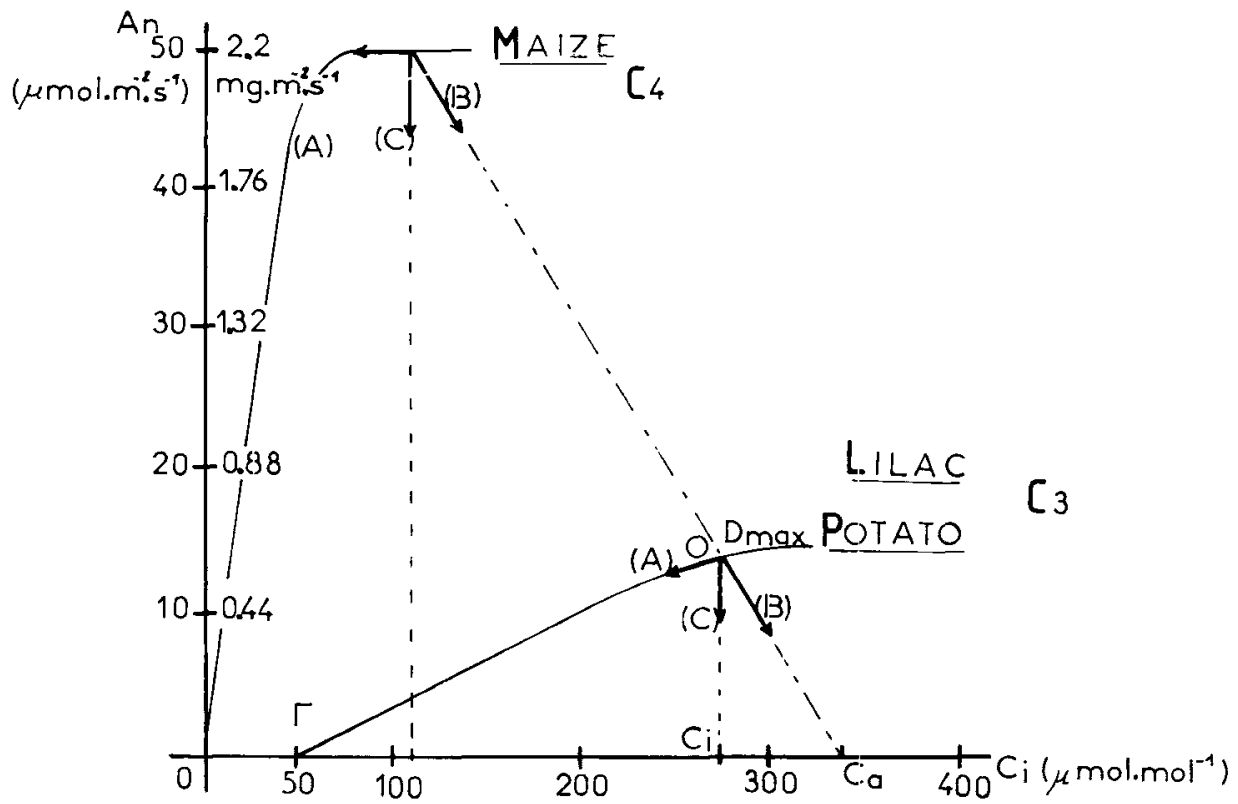

Fig. 1. Theoretical adjustment between demand and supply functions: 3 hypothetical patterns are displayed: A: supply function shifts without any change in demand function; B: demand function shifts without any change in supply function; $\mathrm{C}$ : regulation: $C_{\mathrm{a}}-C_{\mathrm{i}}=$ constant. 


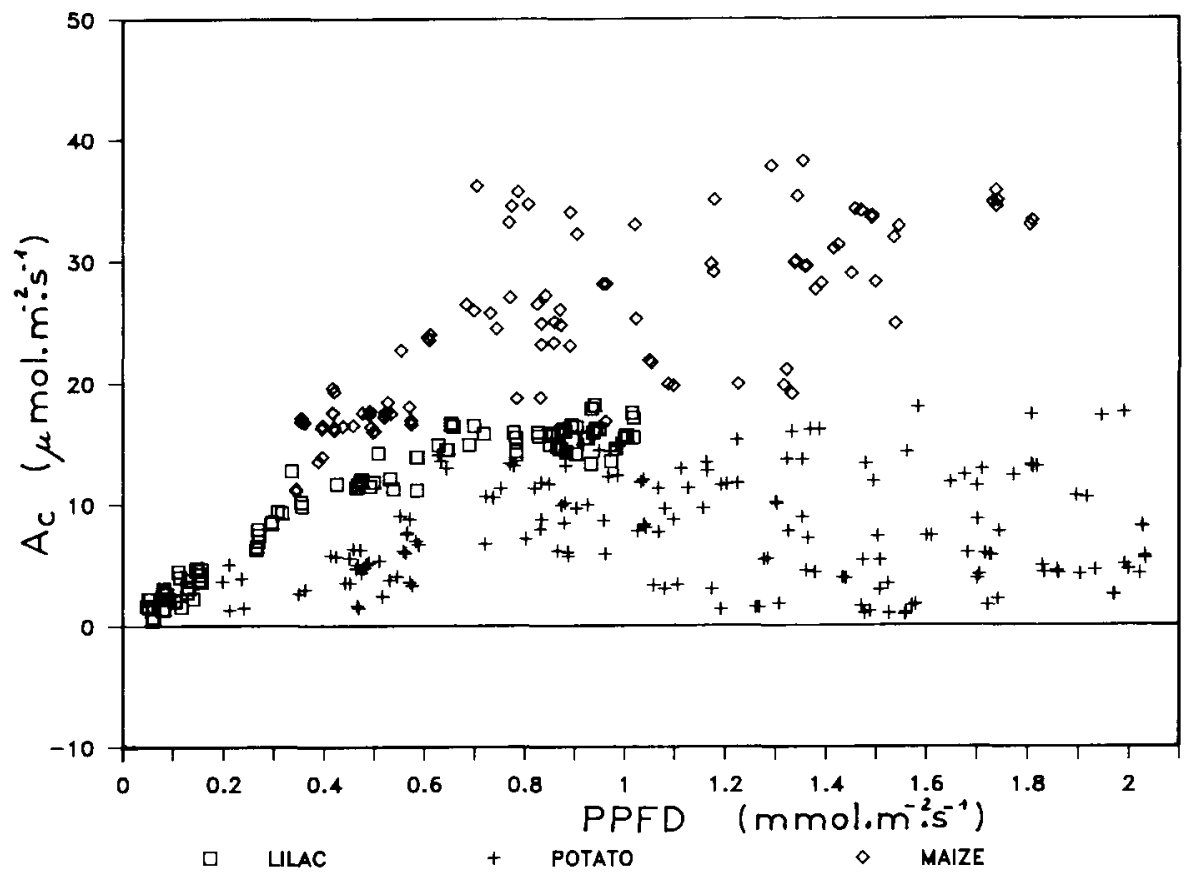

Fig. 2. Net $\mathrm{CO}_{2}$ assimilation $\left(A_{\mathrm{c}}\right)$ versus incident photosynthetic photon flux density, (PPFD); each symbol corresponds to different leaves of a different plant (maize, potato) or a different leaf on 2 trees (lilac). Data are from Aug. 1, 1986 (potato); Aug. 17 (maize), and Sept. 7, 1987 (lilac).

$\mathrm{mol} \cdot \mathrm{m}^{-2} \cdot \mathrm{s}^{-1}$, the $g_{\mathrm{c}}$ dependence of $A_{\mathrm{c}}$ is almost linear and the slope of this line represents $C_{\mathrm{a}}-C_{\mathrm{i}}$. Beyond these values, $A$ does not increase for both $\mathrm{C}_{3}$ plants, although $g_{\mathrm{c}}$ can be large for lilac. Consequently, 2 phases exist in this $A-g_{c}$ relationship: a $C_{i}$ regulated phase for $g_{c}$ below $0.2 \mathrm{~mol} \cdot \mathrm{m}^{-2} \cdot \mathrm{s}^{-1}$, and a maximum assimilation phase for $g_{\mathrm{c}}$ above $0.23 \mathrm{~mol} \cdot \mathrm{m}^{-2} \cdot \mathrm{s}^{-1}$.

\section{Discussion and Conclusion}

The relation between net assimilation $\left(A_{c}\right)$ and leaf conductance to $\mathrm{CO}_{2}\left(g_{\mathrm{c}}\right)$ is de- scribed by a hyperbolic curve (Schulze and Hall, 1982; Küppers, 1984), which may be reduced to both asymptotes (Pereira et al., 1987). The regulated phase and the maximum assimilation phase could be summarized by these 2 asymptotes (Fig. 5). $H$ is the point where the maximum of demand function crosses the $C_{\mathrm{i}}$ regulation line. We can observe that, if WUE of maize is higher than the WUE of lilac or potato, the junction occurs within the same range of values of $g_{\mathrm{c}}$ $\left(0.2 \leq g_{c}<0.23 \mathrm{~mol} \cdot \mathrm{m}^{-2} \cdot \mathrm{s}^{-1}\right)$ for the 3 plants studied here, which are known to be very different from one another as far as $\mathrm{CO}_{2}$ fixation is concerned. Above these values of $g_{c}$, water is wasted. 


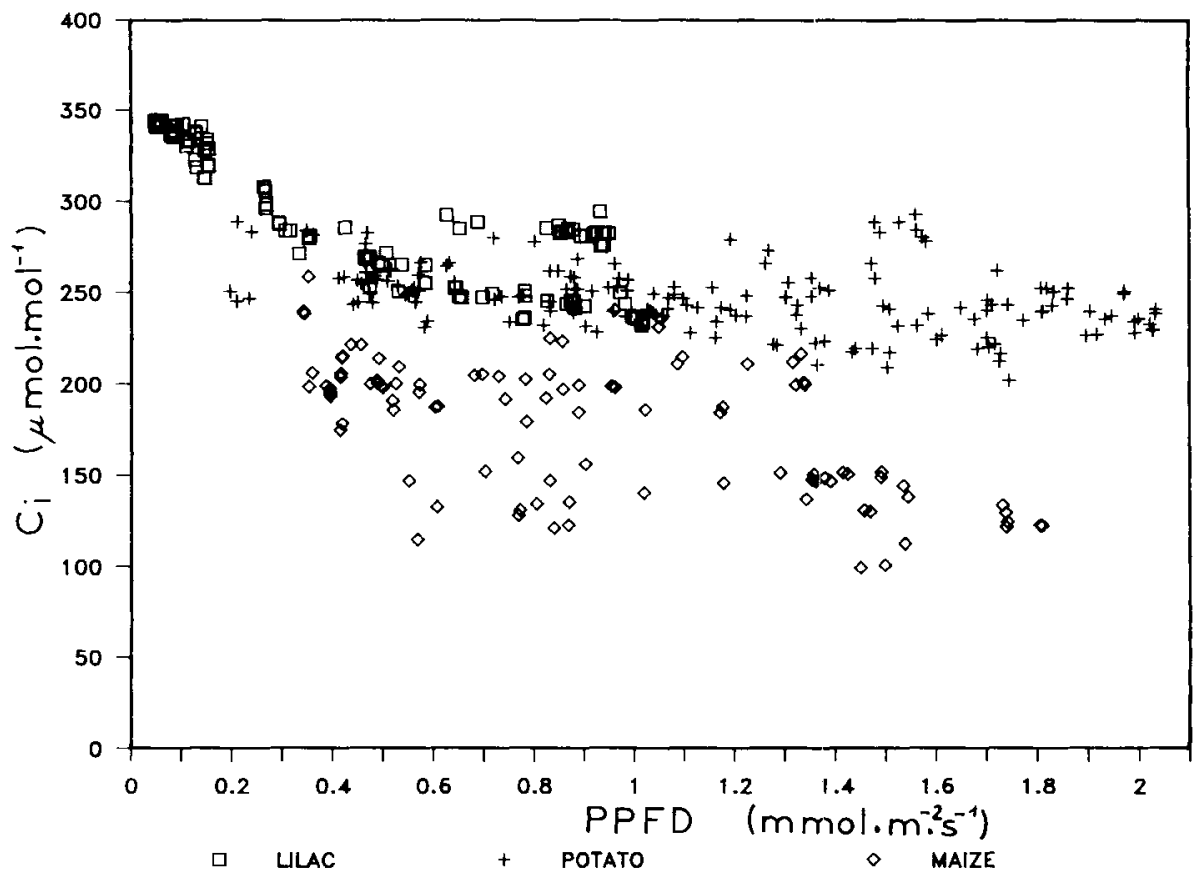

Fig. 3. $C_{\mathrm{i}}$ versus $P P F D$, with the same symbols as those in Fig. 2.

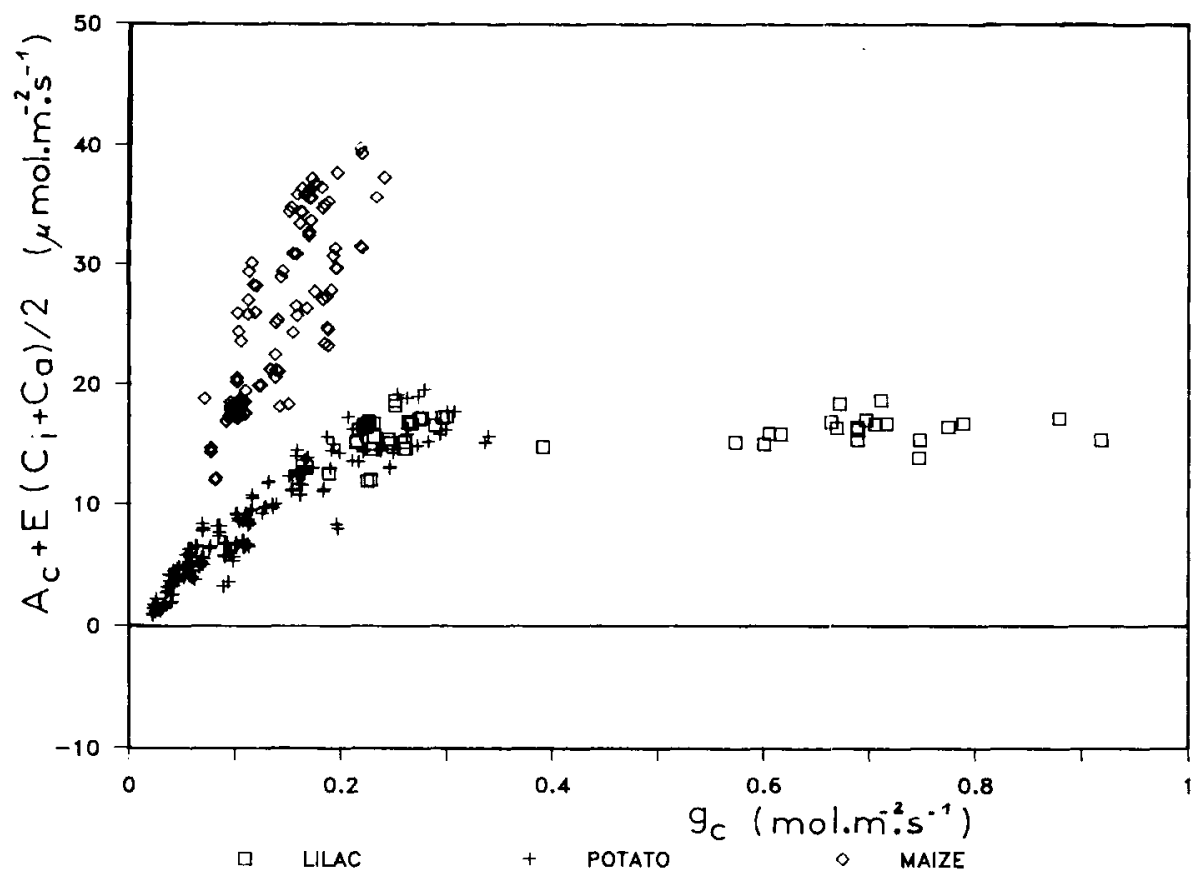

Fig. 4. $A_{c}$, corrected for transpiration efflux, versus $g_{c}$; same symbols as those in Fig. 2 with PPFD $>400$ umol ${ }^{-2} \cdot \mathrm{s}^{-1}$. 


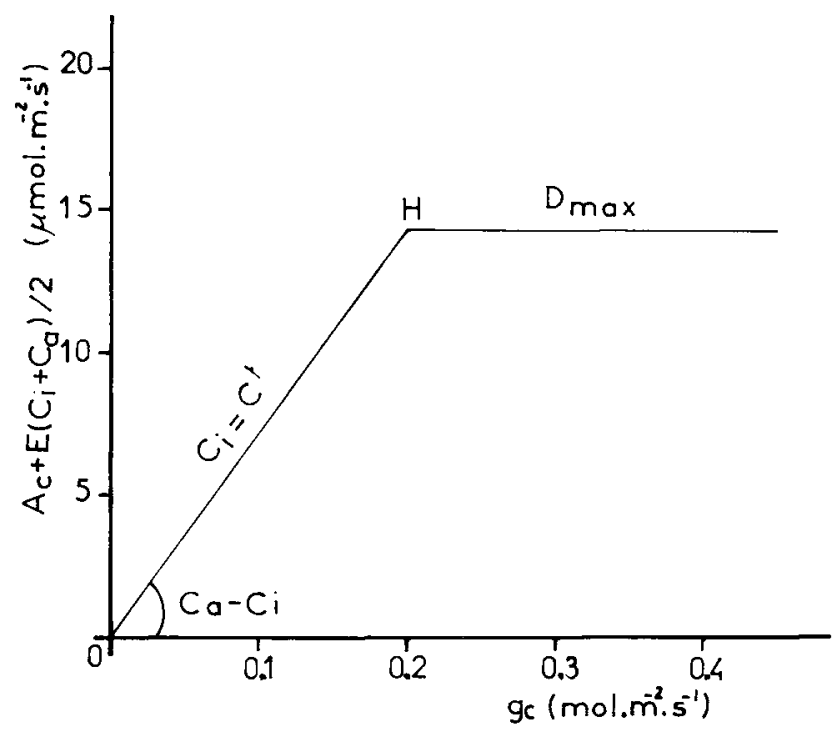

Fig. 5. Ideotype of the relationship between $A_{\mathrm{c}}$ and $g_{\mathrm{c}} . H$ would be the function of the maximum demand function $\left(D_{\max }=A_{\max }\right)$ and the $C_{i}$ regulation line.

\section{References}

Bethenod O., Katerii N., Quetin P. \& Bertolini J.M. (1988) Efficience de l'eau d'une culture de pomme de terre (Solanum tuberosum L. cv. Bintje) 1. Mise en évidence de la régulation du $\mathrm{CO}_{2}$ interne à l'échelle foliaire. Photosynthetica 22, 491-501

Bierhuizen J.F. \& Slatyer R.O. (1965) Effect of atmospheric concentration of water vapor and $\mathrm{CO}_{2}$ in determining transpiration-photosynthesis relationship of cotton leaves. Agric. Meteorol. 2, 259-270

Farquhar G.D. \& Sharkey T.D. (1982) Stomatal conductance and photosynthesis. Annu. Rev. Plant Physiol. 33, 317-345

Jones H.G. (1973) Limiting factors in photosynthesis. New Phytol. 72, 1089-1094

Küppers M. (1984) Carbon relations and competition between woody species in a Central
European hedgerow. II. Stomatal response, water use, and hydraulic conductivity in the root/leaf pathway. Oecologia (Berlin) 64, 344354

Pereira J.S., Tenhunen J.D. \& Lange O.L. (1987) Stomatal control of photosynthesis of Eucalyptus globulus Labill. Trees under field conditions in Portugal. J. Exp. Bot. 195, 16781688

Schulze E.D. \& Hall A.E. (1982) Stomatal responses, water loss and $\mathrm{CO}_{2}$ assimilation rates of plants in contrasting environments. In: Physiological Plant Ecology II: Water Relations and Carbon Assimilation. (Lange et al., eds.) Encyclopedia of Plant Physiology New series, vol. 12B, Springer-Verlag, Berlin, pp. 181-230

Wong S.C., Cowan I.R. \& Farqubar G.D. (1979) Stomatal conductance correlates with photosynthetic capacity. Nature 282, 424-426 